\title{
Condiciones de salud y factores relacionados con discapacidad en adultos mayores -Una reflexión para la atención-
}

\author{
Health conditions and disability-related factors in older adults -A reflection for care-
}

Andrea Patricia Calvo-Soto ${ }^{*}$ orcid.org/0000-0002-1723-9021

Esperanza Gómez-Ramírez¹ orcid.org/0000-0001-7610-244X

1 Grupo de investigación Salud y Movimiento, Programa de Fisioterapia, Facultad de salud, Universidad Santiago de Cali. Cali, Colombia

\begin{abstract}
Resumen
Introducción: Es clara la situación en el mundo del crecimiento de la población adulta mayor. El envejecimiento también trae consigo una carga de importante enfermedad crónica, donde las enfermedades cardiovasculares, de salud mental y el cáncer se ubican entre las prevalentes. Además, se incrementa la necesidad de asistencia de estas personas en el largo plazo porque se pierde la movilidad, aumenta la fragilidad, las secuelas por enfermedades físicas o mentales que aumentan los índices de discapacidad. Objetivo: Reflexionar sobre los aspectos de salud y discapacidad de los adultos mayores según lo reportado en la literatura. Materiales y Métodos: Revisión de literatura de artículos de bases de datos científicas especializadas y repositorios académicos, usando descriptores lógicos y operadores booleanos relacionados con el tema. Resultados: Se estructuró la información teniendo en cuenta los aspectos relacionados con el estado de salud de los adultos mayores, la condición de discapacidad, los factores de riesgo y factores protectores. Conclusiones: Se apoya el diagnóstico temprano de enfermedades prevalentes en los adultos mayores, definiendo los factores de riesgo de discapacidad, pero haciendo hincapié en la intervención con base en el envejecimiento saludable.
\end{abstract}

Palabras clave: Anciano; estado de salud; envejecimiento; personas con discapacidad. (Fuente: DeCS, Bireme).

\begin{abstract}
Introduction: The situation in the world of the growth of the older adult population is clear. Ageing also brings with it a burden of important chronic diseases, where cardiovascular, mental health and cancer diseases are among the prevalent. In addition, the need for assistance from these people in the long term is increased because mobility is lost, fragility increases and physical or mental illnesses sequelae enhance the rates of disability. Objective: To reflect on the aspects of health and disability of older adults as reported in the literature. Materials and methods: Literature review of articles of specialized scientific databases and academic repositories, using logical descriptors and boolean operators related to the subject. Results: The information was structured taking into account the aspects related to the health status of older adults, the condition of disability, risk and protective factors. Conclusions: Early diagnosis of prevalent diseases in older adults is supported by defining the risk factors of disability but emphasizing the intervention based on healthy aging.
\end{abstract}

Key words: Aged; health status; aging; disabled persons. (Source: DeCS, Bireme).

\footnotetext{
*Autor de correspondencia Andrea Patricia Calvo Soto

e-mail: acalvosoto@yahoo.es
} 


\section{Introducción}

El envejecimiento se concibe como un proceso inevitable, que está determinado por factores endógenos y exógenos en el individuo, caracterizado por la degeneración estructural y funcional progresiva de sistemas, órganos y tejidos(1). Es por ello, el interés de determinar cómo la genética, el ambiente, los estilos de vida, entre otros, definen la forma de envejecer. Existen unos componentes fenotípicos del envejecimiento, que incluyen transformaciones estructurales y funcionales que se dividen de manera artificial en cambios primarios del envejecimiento o en enfermedades relacionadas con la edad(2). En ese sentido se conoce que este proceso varía de una persona a otra, lo cual se va a estar reflejado en el estado de salud como en el funcional de los adultos mayores (AM).

En el mundo cada vez se encuentran más AM, de este significativo cambio demográfico son conscientes las instituciones. Según la Organización Mundial de la Salud (OMS) en su informe de 2015 señala que entre 2000 y 2050 , la proporción de personas mayores de 60 años se va a duplicar, llegando al $22 \%$, en números significa que se pasará de 605 millones a 2.000 millones en este periodo de tiempo. Es así como se tendrán más individuos sobrepasando los 80 años, también se proyecta que estos cambios serán más acelerados en países con ingresos bajos y medios(3).

Además del crecimiento poblacional de AM, también debe señalarse que en ellos se presenta un incremento de enfermedades crónicas. La OMS reportó que el número de personas con discapacidad va en aumento, esto debido al envejecimiento de la población, pues las personas ancianas tienen mayor riesgo de discapacidad(4); también se debe al crecimiento global de los problemas crónicos de salud como la diabetes, las enfermedades cardiovasculares y los trastornos mentales. Se estima que las enfermedades crónicas representan el 66\% de todos los años vividos con discapacidad en los países con ingresos bajos y medianos(5). Tener más AM y muchos de ellos con discapacidad, da como resultado mayores niveles de dependencia.
Debido a la problemática previamente descrita se plantea como propósito de este artículo presentar aspectos relacionados con el estado de discapacidad de los AM, según lo reportado en investigaciones en el ámbito mundial, y reflexionar sobre los tópicos a considerar para la atención de esta población.

\section{Materiales y métodos}

El presente artículo reflexivo es producto del trabajo documental titulado: Estado de salud y discapacidad de los AM en América Latina y el Caribe. Una revisión de literatura entre periodo 2010-2017, en la cual se analizaron sesenta documentos (60), de los cuales 44 correspondieron a artículos de revistas científicas indexadas. Los restantes son documentos del Gobierno y tesis que abordaron la temática. La búsqueda se realizó en bases de datos especializadas y repositorios académicos, usando descriptores lógicos y operadores booleanos relacionados con el tema. El presente artículo de reflexión se construyó específicamente, con base en 37 artículos de revistas científicas sobre trabajos desarrollados en los siguientes lugares: México, Brasil, Colombia, Chile, Taiwán, Estados Unidos, Bangladesh, Albania, Canadá, Gran Bretaña, España, Japón, Sudáfrica, China, Noruega, Holanda y Singapur.

\section{Resultados}

Los resultados se presentan en tres apartados principales: la condición de salud de los adultos mayores, la discapacidad y los factores protectores en esta población, en la discusión se expresan aspectos reflexivos relacionados con la atención.

\section{Condiciones de salud de los adultos mayores}

Con respecto a la salud se reportan varias condiciones en los AM, muchas de ellas en relación con enfermedades cardiovasculares, como consecuencia de los padecimientos mayormente autorreportados como la hipertensión, diabetes e hipercolesterolemia(6), esta última representada en la elevación de los niveles séricos de colesterol total, un nivel bajo 
de lipoproteína de alta densidad, con desarrollo de hipertrigliceridemia y obesidad central(7). Igualmente debe mencionarse que pese a los esfuerzos de los entes sanitarios para mejorar la cobertura y la atención, aún existe población de AM con patologías no diagnosticadas. Un estudio reportó que casi la mitad de la muestra tenía presión arterial que se midió como hipertensiva, pero que aún no se había definido como tal con ese diagnóstico, mientras que un tercio autoinformó que un profesional de la salud les había dicho que tenían hipertensión(8). En España, entre el total de altas hospitalarias para esta población están: las enfermedades circulatorias $(21,8 \%)$, respiratorias $(15,0 \%)$, digestivas $(12,3 \%)$ y neoplasias $(11,4 \%)$, como las causas más frecuentes de asistencia sanitaria. Le siguen las lesiones, las enfermedades genitourinarias y las del sistema osteomuscular( ${ }^{(9)}$.

Presentar mayor morbilidad en el envejecimiento se relaciona con el estado funcional futuro del adulto mayor, en esta etapa se presenta disminución de la reserva fisiológica, lo que conlleva a la persona a un importante estado de fragilidad. La fragilidad contempla morbilidad mental y conductual, también en lo neurológico se incluye el accidente cerebrovascular. Sin embargo, dentro de esta condición no solo está presente la afectación al sistema cardiovascular, como regularmente se describe en los AM, porque también existen compromisos en el sistema visual $y$ musculoesquelético(8). Ambos procesos, la fragilidad y las enfermedades crónicas que conllevan a discapacidad pueden cursar de manera independiente, paralela o combinar sus efectos, lo que tiene consecuencias decisivas en la patogenia, curso y pronóstico de la condición(10). Un ejemplo de ello es el seguimiento que se hizo a pacientes prefrágiles y frágiles durante tres años, pues finalizado ese periodo algunos participantes desarrollaron discapacidad o informaron al menos una caída dentro de los 12 meses anteriores(11).

Se precisa que con una mayor presencia de enfermedades crónicas acrecienta la posibilidad de tener discapacidad severa $(p<0,001)$, en comparación con aquellos con una sola enfermedad crónica o sin enfermedad crónica(8). Además de los aspectos físicos y de salud, están las condiciones tempranas y socioeconómicas que afectan el estado funcional, por tanto en los análisis poblacionales sobre prevalencia de discapacidad en AM, existen diferencias entre los países con altos y bajos ingresos, mostrando como la discapacidad en el envejecimiento se constituye en un fenómeno heterogéneo y dependiente de varios aspectos.

Si bien la discapacidad en AM se asocia con secuelas de enfermedades cardiovasculares, también debe considerarse la influencia del sistema musculo esquelético en la movilidad. Mientras en algunos estudios las enfermedades cardiovasculares fueron más prevalentes $(12,13)$, en algunos trabajos lo fue la artritis, seguida de diabetes, ataque cardíaco, accidente cerebrovascular, fractura de cadera y cáncer(14); Entre el grupo de enfermedades diagnosticadas, para la población mayor de 75 años se encuentran: osteoartritis de rodilla, osteoporosis, estenosis del canal espinal, espondilitis espinal(14) y diferentes enfermedades osteodegenerativas(15). Esto deriva al uso de andadores, historial de caídas y de fractura en los últimos años(14), además del dolor consecuente que se relaciona con la reducción en la calidad de vida, descenso en la calidad del sueño, compromiso en el nivel físico y social, ansiedad y depresión(16). Todo lo anterior le da significado al dolor con respecto a la dificultad para la movilidad independiente, sobre todo dependiendo de la ubicación, si es en un sitio (18\%), en sitio múltiple (24\%) o presentando dolor generalizado (39\%).

Ancianos con dolor multisitio o generalizado, tuvieron tres veces mayor riesgo de presentar dificultad inicial de movilidad, en comparación a los participantes sin dolor. El dolor generalizado disminuyó el rendimiento de la movilidad, se encontraron asociaciones similares en cuanto a la interferencia por dolor inicial, el cual va a predecir disminución de la movilidad y posterior discapacidad(17). Esto da pertinencia a la intervención fundada en actividad física y ejercicio, pues más allá de los cambios morfofisiológicos del sistema musculoesquelético 
que suceden durante el envejecimiento, estos se constituyen en estrategias de mantenimiento de las cualidades físicas y de la función. En los aspectos de salud, no solo se señalan cambios en el componente físico relacionados con la funcionalidad y movilidad, pues durante esa etapa también aparecen cambios en la esfera mental a causa de síntomas depresivos, deterioro cognitivo y demencia(6).

Dentro los procesos de salud y discapacidad cabe señalar que estos no son lineales ni están relacionados solamente con la edad, pues existe información que los relaciona con las condiciones tempranas de pobreza, esas condiciones incluyen: aspectos nutricionales intrauterinos y perinatales, incluyendo el crecimiento y el desarrollo en la primera infancia e infancia, padecimiento de enfermedades infecciosas y parasitarias, experiencia de entornos estresantes y otros aspectos asociados con las condiciones socioeconómicas de la familia, todas estas características aumentan el riesgo de sufrir enfermedades crónicas relacionadas con discapacidad. Es tan fuerte la influencia de este aspecto, que las malas condiciones tempranas aumentan las probabilidades de padecer varias comorbilidades(18). Reconociendo este impacto, es claro el riesgo de ser discapacitado para las poblaciones ancianas en América Latina y el Caribe, en comparación con otras latitudes donde las situaciones de pobreza están más superadas.

\section{Discapacidad en adultos mayores}

Para iniciar, se señala que los instrumentos para medir la discapacidad en AM, en los artículos que se revisaron fueron variados y con diversas dimensiones a evaluar, entre ellos estuvieron: el WHODAS 2.0, la batería Katz, cuestionario medición de discapacidad del grupo de Washington, encuestas internacionales, evaluaciones específicas del rendimiento físico, equilibrio, marcha, probabilidad de caídas, evaluación funcional del adulto mayor, cuestionario de salud de Stanford, cuestionarios propios, cuestionario de función locomotriz geriátrica y autoreportes de discapacidad; muchas de esas baterías no tienen la especificidad de aplicación con población AM. Gran parte de trabajos consultados están basados en auto informes de discapacidad para la realización de actividades de la vida diaria (AVD) e instrumentales (AVDI), muy pocos sobre medidas objetivas de discapacidad individual, aunque no se puede desconocer el ejercicio de entes internacionales como el National Health Institute (NHI) y la OMS al introducir preguntas y cuestionarios de medición con características más homogéneas, que permiten comparar poblaciones.

Por otro lado, se informan discrepancias en los diferentes niveles de discapacidad relacionadas con el sexo de los AM, por ejemplo se ha encontrado mayores niveles de discapacidad en mujeres en comparación con hombres. Hubo diferencias de género en todas las discapacidades, excepto en la discapacidad para el recuerdo(18). La probabilidad de tener discapacidad funcional fue mayor para las mujeres que para los hombres. Sin embargo, tanto los hombres como las mujeres que sufrían de una condición crónica estaban en peor situación, en términos de discapacidad funcional y de autocuidado, que aquellos que estaban libres de cualquier condición crónica. Adicionalmente, las probabilidades de tener discapacidad de autocuidado aumentaron en la medida en que la edad era más avanzada(19). De manera similar se presentan los resultados en investigaciones de género, pues los participantes con roles femeninos de género tenían prevalencias de discapacidad de movilidad más altas, en comparación con los que presentaban roles andróginos(20).

Al analizar según el sexo la relación existente entre las variables que corresponden a medidas de higiene, se observa que las mujeres podían realizar estas acciones por sí mismas, por ejemplo bañarse solas; mientras que en los hombres se obtuvo un porcentaje menor y esta diferencia fue estadísticamente significativa(21). Pese a que en las mujeres mayores se encontró mayor prevalencia de discapacidad, en ellas hubo mayor independencia para las actividades instrumentales. El 89,8\% de las mujeres pudo tomar sus medicamentos por sí mismas, 
mientras que en los hombres este porcentaje fue menor. De las mujeres, aproximadamente el $84,4 \%$ prepara su comida por sí mismas, mientras que el 76,6\% de los hombres lo lleva a cabo. El 88.9\% de las mujeres realiza sus labores domésticas por sí mismas, en contraste con el $61,4 \%$ de los hombres. En lo que se refiere al manejo del dinero, el 99,6\% de las mujeres son independientes en contraste con el 67,4\% de hombres, esta diferencia fue estadísticamente significativa ${ }^{(22) .}$

Se ha mencionado que tener un diagnóstico comórbido de enfermedad crónica, aumenta las probabilidades de tener puntuaciones más altas de discapacidad. Las enfermedades crónicas que se asociaron significativamente con discapacidad fueron: los problemas cardíacos, el accidente cerebrovascular $y$ el ataque isquémico transitorio. En contraste existen, reportes que mencionan que aquellos con hipertensión diagnosticada o aquellos con hipertensión arterial que no habían sido diagnosticados, no tuvieron probabilidades significativamente más altas de estar severamente discapacitados, en comparación con aquellos que no tenían hipertensión(8).

La artritis limita significativamente cualquier AVD, como subir y bajar escaleras, caminar media milla, mientras que la diabetes genera una limitación significativa para la mayoría de los indicadores excepto bañarse, arreglarse personalmente, comer y usar el baño. El ataque al corazón estuvo menos asociado con la función de movilidad total y solo fue significativo para subir y bajar escaleras, y caminar media milla. Finalmente, la obesidad emerge como una posible explicación de la discapacidad autoevaluada pues se asoció significativamente con caminar a través de una habitación pequeña, con limitación en las AVD, subir escaleras y caminar media milla(23).

Para los casos de síndrome metabólico, la obesidad central se encontró fuertemente asociada con alto nivel predicho de discapacidad en las AVD, AVDI, movilidad de miembro inferior y movilidad general. Altos niveles de triglicéridos estuvieron significativamente asociados con discapacidad de la vida diaria, instrumental, movilidad de la extremidad inferior, actividades sociales y el ocio, así como con la movilidad general.

En las mujeres entre mayores componentes del síndrome metabólico tuvieran, se presentaba un incremento en la discapacidad total predicha. Para hombres y mujeres la obesidad abdominal e hipertrigliceridemia estuvo significativamente asociada con alta discapacidad predicha(7). Un ejemplo está en Argentina, en donde se establece gran cantidad de población envejecida en Latinoamérica, donde las prevalencias de discapacidad y de obesidad fueron mayores para las personas de mayor edad (65 años y más) respecto al grupo más joven (18 a 64 años) en comparación con la situación en otros países de la región(24). Estos hallazgos se contrastan con la información obtenida en Costa Rica, donde la prevalencia de obesidad es bastante mayor en las mujeres pero tiende a ser menor en las personas de más edad, dada la pérdida de peso de los individuos conforme envejecen, en tanto el bajo peso es un factor de riesgo de sobrevida, constituyéndose la grasa corporal y abdominal en factores protectores(25).

En algunos casos, la obesidad también se comporta como un mecanismo protector para la discapacidad, porque entre los principales factores de riesgo de discapacidad en los AM están las caídas y fracturas, estas últimas se corresponden con la presencia de osteoporosis, patología que tiene menor prevalencia entre las personas mayores con elevados niveles del índice de masa corporal (IMC)(24,26). Así que el sobrepeso para la discapacidad en personas mayores debe mirarse con cautela, pues si bien tiene elementos de riesgo para la salud cardiovascular, para el sistema musculoesquelético se torna en una influencia protectora de enfermedad y discapacidad consecuente.

Es así como con cada aumento en el número de enfermedades crónicas reportadas, hay un incremento en la probabilidad de estar severamente discapacitado, principalmente entre aquellos más pobres(8). Este elemento de pobreza impactaría sobre la discapacidad en AM, 
incluso por encima de las enfermedades que se padecen, pues se encuentra relación entre las condiciones socioeconómicas tempranas y los indicadores de desarrollo temprano (el peso al nacer, el crecimiento físico, las habilidades motrices avanzadas y cognitivas en la infancia, etc.), cuya consecuencia es independiente de las enfermedades crónicas(18).

AM que vivían en la comunidad y con dolor crónico en múltiples ubicaciones musculoesqueléticas, tenían un riesgo sustancialmente mayor de desarrollar discapacidad a lo largo del tiempo, así como un declive clínicamente significativo en el rendimiento de la movilidad(17). La fractura de cadera se asoció con la necesidad de asistencia en las AVD (pasar de una cama a silla, caminar a través de una habitación pequeña, vestirse, usar un inodoro, para el aseo y para comer). La edad se asoció significativamente con todas las actividades como: el baño, subir y bajar escaleras y caminar media milla(23).

La depresión y la demencia también estuvieron fuertemente asociadas con discapacidad(27). Tener problemas con la vista fue la discapacidad más reportada, seguida de limitación al caminar, escuchar, recordar, cuidarse a uno mismo y comunicarse. La dependencia funcional es uno de los problemas más graves de los AM, pues dificulta la atención y el acceso a los servicios de salud, y es particularmente disruptiva cuando se acompaña de deterioro cognitivo(6).

Se encontró mayor prevalencia de discapacidad general, calificada como moderada, principalmente para los dominios funcionales, en comparación con la discapacidad grave, esta última presente en el autocuidado(20). En algunos estudios se presentaron mayores dificultades para las AVD que para las AVDI, en los ancianos estas dificultades se incrementan con la edad y es mayor en las mujeres ${ }^{(6)}$. Esto se corrobora con lo encontrado en otros trabajos, donde es evidente la jerarquía del declive funcional, pues las tareas de movilidad fueron más desafiantes que las AVDI y las de AVD. Sin embargo, las instrumentales no fueron claramente más desafiantes que las AVD (por ejemplo, "manejar dinero" o "preparar una comida caliente")(11). Del mismo modo, la localización de elementos para actividades aparentemente similares (por ejemplo, "caminar 100 metros" y "caminar por una habitación") fueron marcadamente diferentes(28). En el análisis de los resultados, se detectó que los AM evaluados realizaban de manera independiente las medidas de higiene personal (bañarse sin ayuda, lavarse las manos y los pies); con relación a la toma de sus medicamentos, el 88,6\% lo realizaba sin ayuda, preparaba su comida el $73,6 \%$ de manera autónoma, el 85,5\% manejaba su propio dinero y el $70 \%$ hacía sus propias tareas de la casa. El $82 \%$ de los AM fueron independientes y el $18 \%$ estuvo en riesgo de dependencia, desde el punto de vista de funcionalidad, predominó la independencia(22).

La discapacidad previa y la edad son los predictores más fuertes para la futura discapacidad en personas mayores. El sexo femenino, el funcionamiento cognitivo, la autoevaluación de la salud se destacaron como variables importantes en comparación con el grupo control; la obesidad, la hipertensión y las quejas articulares contribuyeron al aumento de la discapacidad, pero rindieron puntuaciones relativamente bajas $^{(22)}$. Los participantes con bajo nivel educativo (sin escolaridad) tenían una mayor prevalencia de discapacidad mental $(p=0,04)$. En un estudio realizado en la zona rural de Bangradesh en general, la prevalencia de discapacidad visual, dificultades auditivas y dificultades de movimiento fueron, respectivamente, más altas entre mujeres que hombres. La prevalencia de dificultades auditivas y de movimiento fueron más altas entre los fumadores en comparación con los no fumadores(29).

Para los trastornos locomotores se reportaron mayores dificultades en las actividades básicas e instrumentales, mostraron relaciones significativas en el grado de discapacidad con el número y la gravedad de la limitación de la actividad. Los hallazgos sugieren que la agravación del nivel de discapacidad en las AVD, se asocia con el aumento en el número de limitaciones y dificultad para ejecutar las 


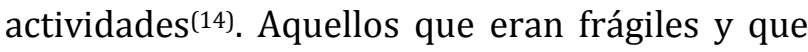
tenían más características relacionadas con la fragilidad, tenían el doble de probabilidades de experimentar restricción de participación que aquellos que eran pre-frágiles(30).

En la prueba Timed up and go -TUG- (se mide como el tiempo -en segundos- que tarda un participante en levantarse de una silla-con reposabrazos-, caminar tres metros de manera rápida pero segura, girar y caminar de regreso a la silla y sentase), para aquellos que les tomó un tiempo mayor para completar la prueba tenían 1,21 veces más probabilidades de tener restricciones de participación, que aquellos que podían terminar la prueba en menor tiempo. Quienes exhibieron más síntomas de depresión, tenían peor calidad de sueño y estaban más preocupados por una caída, además tenían un estatus social autopercibido más bajo y un nivel de actividad física inferior, por tanto tenían más probabilidades de tener restricciones de participación(31). Los que informaron mala salud tenían una puntuación TUG más prolongada, mientras que quienes informaron estar muy bien salud desarrollaron la prueba en menor tiempo. Participantes con un índice de masa corporal de $30 \mathrm{~kg} / \mathrm{m}^{2}$ o superior tenía el peor desempeño de la TUG, comparado con los otros grupos.

Por otro lado, hubo una asociación significativa entre la calificación de la TUG y mortalidad. La salud autoinformada es el predictor más fuerte que la TUG con respecto a la mortalidad, pero la TUG agrega valor predictivo, que ya contiene autoinformación de salud. En este estudio en población mayor de 65 años seguidos durante un período máximo de 11,8 años, se encontró evidencia sólida de una asociación entre la puntuación de la TUG pobre y el aumento de la mortalidad por todas las causas, que fue igualmente fuerte en hombres y mujeres. Las razones por las cuales la TUG es predictivo de mortalidad podrían reflejar malestar subyacente y enfermedad crónica. Por ejemplo, en AM la velocidad de la marcha más lenta se asocia con enfermedad cerebrovascular subclínica, incluso entre quienes tienen aparentemente alto funcionamiento. La velocidad al caminar puede capturar cambios biológicos subyacentes relacionados con la edad, así como también enfermedades diagnosticadas y no diagnosticadas(30).

Se mencionan diferencias entre latitudes, pero incluso para diversos países en Latinoamérica existen comportamientos variados en las limitaciones funcionales en los $\mathrm{AM}$, se puede observar que la población de 60 años y más de Buenos Aires reportó menor prevalencia de discapacidades en AVD (17,36\%), seguida de Montevideo $(17,42 \%)$ y La Habana $(19,04 \%)$, mientras que San Pablo $(19,28 \%)$, México $(19,40 \%)$ y Santiago de Chile $(22 \%)$ presentaron las mayores prevalencias. Los autores de esta investigación se preguntan si la menor prevalencia observada en Buenos Aires y Montevideo se debe a mejores condiciones de salud de las personas mayores, a factores contextuales o a percepciones subjetivas más positivas sobre el propio estado funcional(32).

\section{Otros factores relacionados con la discapacidad}

Otros factores relacionados con discapacidad en AM son el número de visitas al médico en los últimos 12 meses, En España de todas las altas hospitalarias el 43,3\% corresponden a las de AM y estos tuvieron estancias más largas que el resto de la población. Más de la mitad de todas las estancias largas en hospitales se debió a población mayor, por ejemplo en el 2014, constituyeron un 54,9\%(9). En cuanto a las hospitalizaciones, un estudio de Brasil mostró una fuerte asociación con la limitación funcional, tanto entre los usuarios del sistema público para una o dos consultas médicas, como para aquellos del sistema privado. La limitación funcional también mostró una fuerte asociación con la aparición de una o más hospitalizaciones. Los resultados de este estudio mostraron que los AM con limitaciones funcionales realizan más visitas al médico y eran más propensos a las hospitalizaciones, independientemente de los factores predisponentes, como la edad y el sexo, los factores habilitantes entre tanto fueron: vivir,

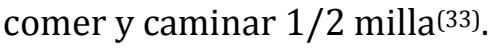

Según un estudio realizado en Bangladesh las probabilidades de tener una discapacidad de 
autocuidado aumentaron en las mujeres AM de familias adineradas, que para las mujeres de familias pobres; tanto las hombres y mujeres de las zonas rurales, tenían mayores índices de discapacidad de autocuidado que sus contrapartes de las áreas urbanas(19). Los factores independientes asociados con la discapacidad de la movilidad fueron ser mujer, soltera, tener menor educación, ingreso insuficiente y la ubicación donde se realizó el estudio(20). En cuanto al aspecto socioeconómico en un estudio realizado en Chile con AM, se presentó mayor prevalencia de deterioro cognitivo ( $p<0,001)$, mala autoevaluación de la salud $(\mathrm{p}<0,001)$ y síntomas depresivos ( $\mathrm{p}<$ 0,001 ), en personas del nivel socioeconómico bajo en comparación con los niveles medio y alto, en este sector bajo también se encontraron peores condiciones para el mantenimiento y recuperación de la limitación, encontrándose así una relación inversa entre el nivel socioeconómico y la limitación funcional; los AM más pobres y menos educados tuvieron más probabilidades de limitación funcional y de muerte(34).

En cuanto a los sujetos con discapacidad severa/extrema en las funciones mentales en España, estos tuvieron un gasto de energía semanal un $62 \%$ más bajo, que los que no tuvieron deterioro o si era leve, hubo también una tendencia similar para las funciones neuromusculoesqueléticas y de movimiento (un $50 \%$ menos de gasto). En la misma dirección, aunque menos marcada, se observó una relación idéntica para la función genitourinaria $y$ reproductiva. Finalmente, las personas con niveles severos a extremos de discapacidad (según WHODAS 2.0), se ubicaron cerca de los que mostraron mayores puntuaciones en el deterioro del funcionamiento mental. Los hallazgos sugieren que las múltiples condiciones médicas pueden conducir a detrimento mental(35).

En poblaciones de Latinoamerica y del Caribe, la discapacidad medida con WHODAS 2.0 se asoció con los niveles educativos, por ejemplo, los participantes que eran amas de casa o retirados tenía puntajes significativamente más altos de
WHODAS, que los participantes que estaban empleados y desempleados. Aquellos que tenían déficits en la cognición tenían más probabilidades de tener niveles más altos de discapacidad. Participantes viudos tenían más probabilidades de tener puntuaciones más altas en WHODAS, versus aquellos que nunca estuvieron casados. La edad se correlacionó positivamente con la gravedad en puntajes de discapacidad para participantes de 85 años o más, quienes informaron niveles más altos de discapacidad, en comparación con aquellos en los grupos de edad de 60 a 74 y de 75 a 84 años. Para finalizar, las probabilidades de tener una limitación funcional fueron más mayores si se es mujer, se fuma y si el nivel de escolaridad es menor(18).

Tanto en países desarrollados como en desarrollo, el contexto social juega un rol fundamental en las limitaciones funcionales en el adulto mayor. Trabajos previos han encontrado que las poblaciones socioeconómicamente más desaventajadas presentan bajos niveles de dependencia funcional. En este sentido, es posible encontrar mayor proporción de personas mayores con dependencia funcional en México y Argentina con respecto a España(36), esto puede estar relacionado con malas condiciones de vida que repercuten en el deterioro de la salud y, consecuentemente, en la dependencia funcional de los AM.

\section{Factores protectores}

Como se señaló en entrevistas realizadas a AM de Brasil, muchos se ven a sí mismos enfermos, y ven la vejez como una tragedia, trayéndoles a ellos ruindad. Esta visión de la vejez se muestra como una tragedia personal, irreversible e irremediable, experimentando un cuerpo quieto e inerte, como muerto(37). Debe exponerse que la percepción de ser viejo, además de los cambios corporales tiene una importante carga social, porque a sociedad es quien le da valor a esos estados. Algunos AM entrevistados, señalaron su estado físico y nivel de movilidad es una elección personal como "quedarse o no quedarse quieto ", esto se relaciona con el cuidado a la salud y el contexto sociocultural(37). 
En algunos trabajos los AM identificados con restricciones de participación eran los más frágiles; aquellos que eran más débiles padecían más enfermedades y tenían un mayor nivel de comorbilidad, además tenían un estatus socioeconómico autopercibido más bajo y menores redes sociales(31). Constituyendo la condición socioeconómica como un factor facilitador en la autopercepción, pues población con mejores condiciones socioeconómicas relataban enfermedades incapacitantes, pero era un discurso más positivo y menos quejante en la vejez(37). El fácil acceso a los servicios sociales y de salud fue un factor de protección independiente que redujo el riesgo de discapacidad y dependencia. Finalmente, vivir en áreas urbanas fue un factor protector contra la discapacidad auto reportada y un factor de riesgo para la dependencia en las $\operatorname{AVD}^{(38)}$.

En Latinoamérica, la población de AM de México encabeza las mayores prevalencias de dependencia, Argentina se ubica en el segundo lugar, en tanto España presenta el valor más bajo. En los tres países se observan magnitudes de personas totales y prevalencias de dependencia muy superiores en las mujeres que en los varones. En el caso de España y de Argentina, la magnitud de mujeres en situación de dependencia más duplica el número de varones con dicha condición, mientras que en México las mujeres en situación de dependencia son en torno a un $60 \%$ más que los varones(36). Esto muestra que un factor protector son las condiciones socioeconómicas del entorno, en este caso contar con una situación favorable en el país donde se crece y/o envejece.

Se encuentran varios factores protectores para hacerle frente a la discapacidad, pues no es una condición inalterable, entre ellos están la actividad física y la participación social, que han sido reportadas con carácter diferencial para hombres y mujeres, por ejemplo las actividades como jugar golf correr o trotar, calistenia y natación. Esta diferenciación por sexo aplica también para el deterioro funcional en las funciones genitourinarias y reproductivas, así como en las funciones de voz y habla, digestivas y endocrinas y funciones neuromusculoesqueléticas y de movimiento(23,35). Para algunos AM; la manera de hacer frente a la funcionalidad es opuesta, con una idea de mantenerse activo, al señalar que el "viejo no puede parar". Sin embargo, esta concepción se revela un cuchillo de dos filos: por un lado, aquietarse podría favorecer la resiliencia de las personas con limitaciones, y por otro, se muestra una estrategia ineficaz y contraproducente de cuidado, la cual, posiblemente, los hará aún más dependientes y desamparados en el futuro(27,37).

Los antecedentes educativos superiores actúan como una medida de protección contra la discapacidad, el deterioro de la cognición influye fuertemente en su desarrollo. Los estudios sugieren que los déficits en varios dominios de la cognición (atención, memoria, el lenguaje y el rendimiento visoespacial) reducen directamente la capacidad de un individuo para realizar tareas cotidianas; la presencia de cualquier enfermedad crónica estuvo fuertemente asociada con discapacidad en esta población(27). En España, los niveles medio y alto de comprensión fueron altamente protectores contra la discapacidad y la dependencia. La frecuencia de los contactos sociales siguió siendo un predictor protector no significativo para la discapacidad y la dependencia. El acceso a los recursos sociales mostró un efecto protector contra la discapacidad y la dependencia dependiente de la dosis; los factores ambientales también se asociaron con discapacidad(38).

En hombres AM de Gran Bretaña, las asociaciones con WHODAS 2.0 fueron más débiles en aquellos con compromiso en las funciones mentales o neuromusculoesqueléticas y del movimiento corporal, sugiriendo que una actividad física baja está asociada con deterioro en estas funciones, esto podría canalizarse a través del rendimiento en la actividad y la participación. De hecho, el trabajo, la movilidad y la atención son dimensiones del "Cuestionario de actividad física de Yale", y son tareas o acciones que se superponen con las actividades y la participación en la clasificación, aunque solo las personas frágiles tuvieron un riesgo significativamente mayor de incidentes de caídas(11). 
En un estudio relacionado con la actividad física, la reducción de la discapacidad para la movilidad desde la línea de base hasta los 24 meses, favoreció la mejoría de la función física cuando la

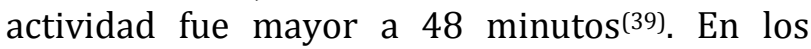
hombres, la cantidad de actividad física de tiempo libre se asoció inversamente con la incidencia de discapacidad. En las mujeres, no se encontraron asociaciones significativas entre la cantidad de actividad física de ocio y la incidencia de discapacidad. Se halló una relación dosis respuesta entre la cantidad de actividad física de tiempo libre, y la incidencia de discapacidad con demencia en hombres, basada en la evaluación cuantitativa de la actividad física; por lo que se puede decir que es aconsejable realizar más actividad física durante el tiempo libre dentro del rango de 18 MET (metabolic equivalent of task) horas /por semana)(40). Sin embargo, no se puede tomar el ejercicio como una sola intervención para reducir la discapacidad, pues hay investigaciones en los que no se encontró evidencia estadísticamente significativa, de que los beneficios de la actividad física reduzcan el riesgo de discapacidad de movilidad y difieran según la educación o el grupo de ingresos. La falta de beneficios diferenciales indica que esta forma de atención, si se establece de forma más amplia, no aumentará ni reducirá el impacto de las desigualdades socioeconómicas en la discapacidad(41), por tanto no debe ser una intervención aislada y descontextualizada a la hora de atender población AM, cuando se tiene el propósito de mejorar sus niveles de funcionalidad.

\section{Discusión}

En el ámbito científico internacional antes de los años 1990 había una concepción de discapacidad centrada en el individuo y sus deficiencias, pero posterior a esa ápoca se sucede una modificación donde se tienen en cuenta los factores contextuales(22,42), esto permite visibilizar la discapacidad y la dependencia en un ámbito que exige acciones al estado, donde se logre modificar el entorno, buscando combatir no solo las consecuencias, sino también las causas de la discapacidad, reduciendo así los niveles de dependencia y el impacto social. En América Latina debe revisarse esta situación, porque en comparación con Europa, su población está envejeciendo más tarde, pero en períodos de tiempo mucho más cortos, con esta aceleración en el ámbito local se espera un crecimiento en los niveles de discapacidad y dependencia(26); sin embargo, en términos de políticas y programas de asistencia a esta población, Europa lleva la delantera, se requiere entonces que en Latinoamérica se agilice el proceso de establecimiento dichas estrategias.

Las enfermedades crónicas en el envejecimiento que se asocian con la discapacidad son las cardiovasculares, relacionadas con funciones de la movilidad, la obesidad con movilidad y AVD, diabetes mellitus con limitaciones para las AVD, las enfermedades musculoesqueléticas con dolor y compromiso de la movilidad (marcha, equilibrio) y los trastornos mentales con discapacidad cognitiva. La discapacidad en AM sucede de manera diferencial para hombres y mujeres, es dependiente del entorno y de las condiciones tempranas de pobreza, en relación a cómo se creció, pero también cómo se ha envejecido, y si el medio actual es facilitador de un mejor escenario socioeconómico que cuente con servicios sociales y sanitarios, esto se resume en el trabajo de Fuentes et al., quienes concluyen al final del seguimiento a una cohorte de AM que quienes tuvieron nivel socioeconómico bajo se mantuvieron limitados y los de un nivel alto fueron funcionalmente sanos, presentando que la incidencia de limitación funcional sigue un gradiente socioeconómico(34). La funcionalidad que se relacionada con la movilidad resultó más desafiante que las AVDI y las AVD, esta última concerniente con las medidas de higiene personal, así como se muestra en otros trabajados, en los cuales la discapacidad física en ancianos presentó un patrón jerárquico, la discapacidad comenzó con dificultad para la movilidad y flexibilidad, luego dificultad para las actividades instrumentadas de la vida diaria y finalmente alteraciones de las $\operatorname{AVD}^{(43)}$.

En cuanto a la información utilizada en este artículo, se presentan unas ideas a reflexionar 
hacia la creciente discapacidad en los AM, una de ellas es que debe mejorarse la cobertura y el diagnóstico temprano de enfermedades prevalentes en AM, cuando estas se ubican en estadios tempranos, considerando que entre más número de patologías crónicas se presenten, mayores niveles de discapacidad se van a encontrar. Al identificar que el número de hospitalizaciones incrementa la posibilidad de desarrollar discapacidad, se justifica en otra medida la razón de realizar atención temprana. Con base en lo anterior, se requiere facilitar el acceso a los servicios de salud para todos los AM, pero especialmente para aquellos con cierto nivel de dependencia, y sobre todo cuando hay déficit cognitivo.

Teniendo en cuenta los factores de riesgo relacionados con la presencia de enfermedades crónicas no trasmisibles, alteraciones neuromusculoesqueléticas y obesidad para el desarrollo de discapacidad futura, se señala que éstas se asocian directamente con los estilos de vida, por ello es importante considerar el envejecimiento como un proceso continuo desde que se nace, en consecuencia las medidas de prevención y adquisición de hábitos saludables, no deben contemplarse solo en la edad adulta, sino siendo unas prácticas que se cultivan desde la infancia y adolescencia. Sí se quiere tener adultos sanos debemos empezar por tener niños sanos, por ello las acciones de promoción deben ser transversales durante el ciclo vital.

Contar con autopercepción positiva, tener altos niveles de escolaridad, desarrollar actividades de ocio y mantener vida social, practicar actividad física y ejercicio, gozar de óptimas condiciones socioeconómicas, vivir en un área rural y tener acceso a servicios sociales son factores protectores de discapacidad en AM. La actividad física, de ocio y la participación social han estado entre las estrategias implementadas en varios gobiernos para hacer frente a la discapacidad en los AM, pero también existen unos aspectos estructurales a atender y en los cuales prevalece la discapacidad en esta población, que son las condiciones socioeconómicas y ubicarse en zona urbana. Esto obliga a que también se desarrollen estrategias diferenciales considerando estos componentes.

Para que la actividad física tenga buenos resultados debe ser regular, con una duración mayor a 45 minutos(39), debe generar un importante gasto energético semanal, incluyendo otras cualidades físicas como el entrenamiento de la fuerza muscular(44), para lograr efectos en la esfera física y mental(45); también se hace necesario contar con contactos sociales y participación, para que luego todo esto se refleje en independencia en la AVD y AVDI, sin descuidar los aspectos diferenciales por género, pues se evidenció que tanto para algunas enfermedades como en la discapacidad, se encuentran discrepancias entre hombres $\mathrm{y}$ mujeres.

Para el caso de los AM, debido al cambio demográfico existe un creciente interés de mejorar las condiciones, desde la perspectiva del bienestar y la salud, pues la discapacidad abarca deficiencias, limitaciones en la actividad y restricciones en la participación, y por ello debe prestarse atención a los factores ambientales y personales. Giacomin y Araujo resaltan la importancia del sistema de salud pública sobre dimensión funcional de la salud en la vejez, por ello es necesario la gestión en todos los niveles (municipios, estados y gobierno federal), los cuales deberían revisar sus conceptos sobre la interacción entre salud, envejecimiento $\mathrm{y}$ discapacidad, para que las políticas públicas de atención por parte del Estado garanticen la naturaleza integral de la atención y la rehabilitación de las condiciones de salud, de una manera interdisciplinaria(46). Todo lo anterior, ofrece un reto para los Ministerios de Salud y Deporte, entre otros, los cuales deben generar las medidas necesarias para que oriente la intervención y la prevención en discapacidad.

\section{Conclusiones}

Es claro que el envejecimiento es un proceso que se presenta en las personas de forma heterogénea, también en cuanto al estado de salud y funcionalidad, expresando que a mayor edad se incrementa la posibilidad de 
dependencia, representando como factores de riesgo para discapacidad, el género, la condición socioeconómica, el nivel educativo, entre otros. Por ello la necesidad de trabajar las políticas y programas dirigidos a esta población sobre los factores protectores, teniendo como base los determinantes del envejecimiento activo que definió la OMS: la cultura y el género, los sistemas sociales y de salud, el comportamiento, los factores personales, el medio ambiente físico y social, y finalmente la economía. Lo anterior en consonancia con las prioridades futuras de envejecimiento saludable, emitidos por entes internacionales, en cuanto al desarrollo de políticas y estrategias, recolección de datos para el monitoreo, promoción de la investigación acerca de las necesidades comunes y futuras de la población de AM, desarrollo de sistemas de salud integrados al cuidado de las personas mayores, implementación de sistemas de cuidado a largo plazo, asegurando los recursos humanos para el cuidado integral, atender a nivel mundial la discriminación por edad, entender los costos y oportunidades el envejecimiento saludable, mejorar la red de ciudades y comunidades amigables con el envejecimiento.

\section{Conflicto de intereses: Ninguno.}

\section{Referencias}

1. Martínez A., Gil Romero L., Serrano P., Ramos J. Nuevas miradas sobre el envejecimiento. 1a ed. Madrid-España: Instituto de Mayores y Servicios Sociales; 2009. $471 \mathrm{p}$.

2. Kasper D, Fauci A, Hauser S, Longo D, Jameson L, Loscalzo J. Principios de Medicina Interna. 19a ed. Vol. 1. New York: Mc Graw-Hill; 2016.

3. Organización Mundial de la Salud. Informe Mundial sobre el envejecimiento y la salud [Internet]. Ginebra: OMS; $2015 . \quad$ Disponible en: http://www.who.int/ageing/about/fSacts/es/

4. Organización Mundial de la Salud. Informe mundial sobre la discapacidad [Internet]. Ginebra: OMS; 2011. Disponible

en: http://www.who.int/disabilities/world_report/2011/e s/

5. Ministerio de Salud y Protección Social. Informe al congreso de la república 2014 - 2015. Sector administrativo de salud y protección social [Internet]. Bogotá: MinSalud; 2015. Disponible en: https://www.minsalud.gov.co/sites/rid/Lists/Bibliotec aDigital/RIDE/DE/PES/informe-congreso-20142015.pdf
6. Salinas A, Moreno K, Acosta I, Sosa A, Gutiérrez L, Téllez M. Condiciones de salud y estado funcional de los adultos mayores en México. Salud Pública México. 2013;55:S323-S331.

7. Fang-Yih, Liaw Tung-Wei Kao, Li-WeiWu, Chung-Ching Wang, Hui-FangYang, Tao-Chun Peng, et al. Components of Metabolic Syndrome and the Risk of Disability among the Elderly Population. Sci Rep. 2016; 6(22750):1-9.

8. Waterhouse P, Van der Wielen N, Chirwa P, Amos A. The impact of multi-morbidity on disability among older adults in South Africa: do hypertension and sociodemographic characteristics matter?. Int J Equity Health. 2017;16:62.

9. Abellán A, Pujol R. Un perfil de las personas mayores en España, 2016. Indicadores estadísticos básicos. [Internet]. Madrid: Informes Envejecimiento en red no 14; $2016 . \quad$ Disponible en: http://digital.csic.es/bitstream/10261/128087/1/enr ed-indicadoresbasicos16.pdf

10. Romero C, Angel T. Fragilidad y enfermedades crónicas en los adultos mayores. Med Interna México. 2011;27(5):455-62.

11. Papachristou E, Wannamethee SG, Lennon LT, Papacosta O, Whincup PH, Iliffe S, et al. Ability of SelfReported Frailty Components to Predict Incident Disability, Falls, and All-Cause Mortality: Results From a Population-Based Study of Older British Men. J Am Med Dir Assoc. 2017;18:152-157.

12. Casas P, et al. Perfil clínico del adulto mayor atendido ambulatoriamente en un hospital general. Rev Medica Hered. 2012;23(4):229-234.

13. Varela L, et al. Perfil clínico, funcional y sociofamiliar del adulto mayor de la comunidad en un distrito de Lima, Perú. Rev Peru Med Exp Salud Pública. 2015; 32(4):709-716.

14. Iwaya T, Doi T, Seichi A, Hoshino Y, Ogata T, Akai M. Characteristics of disability in activity of daily living in elderly people associated with locomotive disorders. BMC Geriatr. 2017;11:165-178.

15. Guerrero R, et al. Perfil gerontológico del adulto mayor en Tabasco, México. Rev Salud Pública. 2012; 14:88101.

16. Navarro N.et al. Prevalencia de alteraciones en la calidad del sueño en adultos mayores con dolor crónico musculoesquelético. Rev Mex Anestesiol. 2015;38(2):79-84.

17. Eggermon L, Leveille S, Shi L, Kiely D, Shmerling R, Jones R, et al. Pain Characteristics Associated With the Onset of Disability in Older Adults: The MOBILIZE Boston Study. J Am Geriatr Soc. 2014;62:1007-1016.

18. Monteverde M, Norohna K, Palloni A. Effect of Early Conditions on Disability among Elderly in LatinAmerica and the Caribbean. Popul Stud. 63(1):21-35.

19. Tareque M, Tiedt A, Islam T, Begum S, Saito Y. Gender differences in functional disability and self-care among seniors in Bangladesh. BMC Geriatr. 2017;17:177-89.

20. Ahmed T, Vafaei A, Auais M, Guralnik J, Zunzunegui M. Gender Roles and Physical Function in Older Adults: Cross-Sectional Analysis of the International Mobility in 
Aging Study (IMIAS). PLoS ONE. 2016;11(0156828):118.

21. Monroy A, Contreras M, García L, García M, CárdenasRivero L. Estatus funcional de adultos mayores de Tláhuac, Ciudad de México. Enferm Univ. 2016;13:2530.

22. Taş Ü, Steyerberg E, Bierma S, Hofman A, Koes B, Verhagen A. Age, gender and disability predict future disability in older people: the Rotterdam Study. BMC Geriatr. 2011;11:22-33.

23. Nam S, Snih S, Markides K. The Effects of chronic medical conditions and obesity on self reported disability in older Mexican American. Tex Public Health J. 2017;69(3):12-5.

24. Monteverde M. Exceso de Peso y Discapacidad de las Personas Mayores en la Argentina. Salud Colect. 11(4):509-521.

25. Peláez E. Sociedad y Adulto Mayor en América LatinaEstudios sobre Envejecimiento en la Región-. Rio de Janeiro, Brasil: ALAP; 2008. (Investigaciones. Primera Edición (segunda parte -envejecimiento y salud).

26. Monteverde M, Tomas S, Acosta L, Garay S. Envejecimiento Poblacional y Magnitud de la Dependencia en Argentina y México: Perspectiva comparada con España. Rev Lat-Am Poblac. 2016;10(18):135-154.

27. Mahesh M, Abdin E, Vaingankar J, Picco L, Jeyagurunathan A, Binte-Shafi S, et al. Disability in Singapore's Elderly Population. Ann Acad Med Singap. 2016;45:284-296.

28. Buz J, Cortés M. Measurement of the severity of disability in community-dwelling adults and older adults: interval-level measures for accurate comparisons in large survey data set. BMJ Open. 2016;6(011842):1-14.

29. Amirul-Islam F, Bhowmik J, Islam S, Renzaho A, Hiller J. Factors Associated with Disability in Rural Bangladesh: Bangladesh Population-Based Diabetes and Eye Study (BPDES). PLoS One. 2016; 11(12):e0165625.

30. Bergland A, Jorgensen L, Emaus N, Heine B. Mobility as a predictor of all-cause mortality in older men and women: 11.8 year follow-up in the Troms $\emptyset$ study. BMC Health Serv Res. 2017;17:22.

31. Yat Wa Liu J. The severity and associated factors of participation restriction among community dwelling frail older people: an application of the International Classification of Functioning, Disability and Health (WHO-ICF). BMC Geriatr. 2017;17:43.

32. Monteverde M, Peranovich A, Zepeda A. Comparación de la prevalencia de discapacidades basada en autoreportes en países de América Latina. Poblac Salud En Mesoamérica. 12(1):1-11.

33. Vaz de Melo J, Viana S, Carvalho D, Lima M. Use of health services by Brazilian older adults with and without functional limitation. Rev Saude Publica. 2017;51(1):1-9.

34. Fuentes A, Sánchez H, Lera L, Cea X, Albala C. Desigualdades socioeconómicas en el proceso de discapacidad en una cohorte de adultos mayores de Santiago de Chile. Gac Sanit. 2013;27:226-232.
35. Caron A, Ayala A, Damián J, Rodríguez C, Almazán J, Castellote J, et al. Physical activity, body functions and disability among middle-aged and older Spanish adult. BMC Geriatr. 2017;17:150-61.

36. Monteverde M, Ramón A, López G. Población mayor en situación de dependencia de la Argentina: Propuesta de clasificación según severidad basada en datos secundarios. Poblac Salud en Mesoamérica. 2017;5(1):1-21.

37. Pereira J, Giacomin K, Oliveira J. A funcionalidade e incapacidade na velhice: ficar ou não ficar quieto. Cad Saúde Pública Rio Jan. 2015;31:1451-1459.

38. Virues J, Vega S, Martínez M, Saz P, Rodríguez F, Rodriguez A, et al. A protective personal factor against disability and dependence in the elderly: an ordinal regression analysis with nine geographically-defined samples from Spain. BMC Geriatr. 2017;17:2-10.

39. Fielding R, Guralnik J, King A, Pahor M, McDermott M, Tudor $\mathrm{C}$, et al. Dose of physical activity, physical functioning and disability risk in mobility-limited older adults: Results from the LIFE study randomized trial. PLoS ONE. 2017;12(0182155):1-14.

40. Matsunaga T, Naito M, Wakai K, Ukawa S, Zhao W, Okabayashi $\mathrm{S}$, et al. Leisure-time physical activity and risk of disability incidence: A 12-year prospective cohort study among young elderly of the same age at baseline. J Epidemiol. 2017;27:538-545.

41. Bann D, Chen H, Bonell C, Glynn N, Fielding R, Manini T, et al. Socioeconomic differences in the benefits of structured physical activity compared with health education on the prevention of major mobility disability in older adults: the LIFE study. J Epidemiol Community Health. 2016;70:930-933.

42. Abellán A, Esparza C, Castejón P, Pérez J. Epidemiología de la discapacidad y la dependencia de la vejez en España. Gac Sanit. 2011; 25:5-11.

43. Lluis G. Fragilidad y asociaciones de riesgo en adultos mayores de una comunidad urbana. Rev Cuba Med Mil. 2013;42(3):368-376.

44. Poblete F, Flores C, Abad A, Díaz E. Funcionalidad, fuerza y calidad de vida en adultos mayores activos de Valdivia. Rev Cienc Act Física. 2015;16(1):45-52.

45. Franco M, Parra E, González F, Bernate M, Solis A. Influencia del ejercicio físico en la prevención del deterioro cognitivo en las personas mayores: revisión sistemática. Rev Neurol. 2013;56(11):545-554.

46. Giacomin K, Araújo J. Old age, disability and care in public health. Ciênc Saúde Coletiva. 2015;20(12):36313640 . 